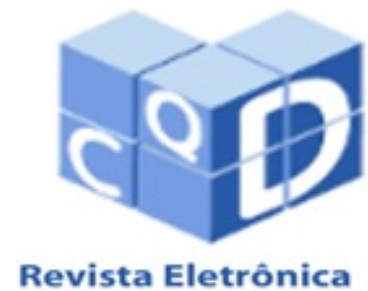

Paulista de Matemática

ISSN 2316-9664

Volume 14, fev. 2019

Edição Ermac

Fernando Santos Silva

Universidade Estadual do

Sudoeste da Bahia

fssilva@uesb.edu.br

Davidson Martins Moreira

Centro Universitário SENAI

CIMATEC

davidson.moreira@gmail.com

\section{Regra da cadeia para derivada fracionária de Caputo com baixo nivel fracionário}

Chain rule for fractional derivative of Caputo type with low-fractionality

\section{Resumo}

Este trabalho apresenta uma aproximação da regra da cadeia da derivada fracionária, do tipo Caputo com baixo nível fracionário, para obter soluções exatas das versões fracionárias das equações diferenciais de Bernoulli e de Riccati.

Palavras-chave: Cálculo Fracionário e Aplicações. Bernoulli. Riccati. Regra da Cadeia. Baixo Nível Fracionário.

Abstract
This work presents an approximation of the Fractional derivative
chain rule of the Caputo type with low fractional level to obtain
exact solutions of the fractional versions of the Bernoulli and Ric-
cati differential equations.
Keywords: Fractional Calculus and Applications. Bernoulli.
Riccati. Chain Rule. Low-Level Fractionality.

\author{
Marcelo Albano Moret \\ Centro Universitário SENAI \\ CIMATEC \\ mamoret@gmail.com
}




\section{Introdução}

O cálculo de ordem não inteira, conhecido como cálculo fracionário, tradução livre de fractional calculus, generaliza o cálculo integral e diferencial clássicos. Pode-se pensar nos operadores de ordem fracionários como os operadores que representam funções da memória sobre a história de alguns sinais de sistemas físicos (CAMARGO; OLIVEIRA, 2015; PODLUBNY, 1999).

Nos últimos anos, a caracterização de sistemas reais por meio de modelos com equações diferenciais de ordem fracionária vem aumentando consideravelmente, pois os mesmos representam com maior fidelidade fenômenos naturais que os modelos de ordem inteira. Mais ainda, em relação ao tempo esses modelos descrevem processos com memória de longo prazo do tipo lei de potência (DU; WANG; HU, 2013; EDELMAN, 2014; JUCHEM NETO; FONSECA, 2018; PODLUBNY, 1999; TARASOV, 2013; TARASOVA; TARASOV, 2017). Desta forma, a evolução do sistema estudado depende de toda a memória dos eventos passados.

Recentemente, foi proposto em Tarasov e Zaslavsky (2006), e de forma independente em Varalta, Gomes e Camargo (2014), casos em que $\alpha$ se desvia minimamente de um valor inteiro $\alpha=n-\varepsilon$. Para $0<\varepsilon<<1$ o comportamento da derivada fracionária é denominado de baixo nível fracionário. Essencialmente, o caso baixo nível fracionário é tradado como uma perturbação para o caso inteiro através da $\varepsilon$-expansão por Tarasov e Zaslavsky (2006). Este método também foi aplicado a oscilação fracionária (TOFIGHI, 2013), fenômenos de relaxamento fracionário (TOFIGHI; GOLESTANI, 2008) e física das partículas (GOLDFAIN, 2008).

Utilizamos o caso de baixo nível fracionário nas versões fracionárias das equações diferenciais dos tipos Bernoulli e de Riccati. Neste caso, podemos aproximar a derivada fracionária de Caputo e utilizar uma versão aproximada da regra da cadeia para obter uma solução semelhante a solução clássica.

Parte deste trabalho foi apresentado durante o Encontro Regional de Matemática Aplicada e Computacional que ocorreu de seis a oito de junho de 2018 na Universidade Estadual Paulista "Júlio de Mesquita Filho" no campus de Bauru.

\section{Preliminares}

Nessa seção introduzimos a definição da derivada fracionária segundo Caputo (1967) e algumas de suas principais propriedades. Maiores detalhes podem ser vistos em Camargo e Oliveira (2015), Diethelm (2010) e Silva, Moreira e Moret (2018b).

Definição 2.1 Sejam $T, \alpha \in \mathbb{R}^{+}$e $n=\min \{k \in \mathbb{N} / k \geq \alpha\}$. A derivada fracionária de ordem $\alpha$ no sentido de Caputo (à esquerda) da função $f \in A C^{n}[0, T]$ é difinida como

$$
{ }_{0}^{C} D_{t}^{\alpha} f(t)= \begin{cases}\frac{1}{\Gamma(n-\alpha)} \int_{0}^{t}(x-s)^{n-1-\alpha} f^{(n)}(s) d s, & n-1<\alpha<n, \\ f^{(n)}(t), & n=\alpha,\end{cases}
$$

onde $f^{(n)}(s)=d^{n} f(s) / d s^{n} e \Gamma(\cdot)$ é a função Gamma. Usaremos a notação simplificada $D_{t}^{\alpha} f(t)=$ ${ }_{0}^{C} D_{t}^{\alpha} f(t)$

Algumas propriedades da derivada de Caputo são bastante semelhantes às da derivada clássica (Newton-Leibniz). A seguir apresentaremos algumas propriedades:

Propriedade $1 D_{t}^{\alpha} K=0$, para toda constante $K$.

SILVA, F. S.; MOREIRA, D. M.; MORET, M. A. Regra da cadeia para derivada fracionária de Caputo com baixo nível fracionário. C.Q.D.- Revista Eletrônica Paulista de Matemática, Bauru, v. 14, p. 147-159, fev. 2019. Edição Ermac. 
Propriedade $2 D_{t}^{\alpha}[\lambda f(t)+g(t)]=\lambda D_{t}^{\alpha} f(t)+D_{t}^{\alpha} g(t)$, onde $\lambda \in \mathbb{R}$ e f,g $\in A C^{n}[0, T]$.

Teorema 2.2 (DIETHELM, 2010) Seja $f(t)=t^{\lambda}$, com $\lambda>0$, então temos a seguinte relação

$$
D_{t}^{\alpha} f(t)=\frac{\Gamma(\lambda+1)}{\Gamma(\lambda+1-\alpha)} t^{\lambda-\alpha}, \quad \lambda>n .
$$

Teorema 2.3 (LI; DENG, 2007) Sejam $f \in C^{n+1}[0, T]$ e $\alpha=n-\varepsilon$, então

(a) $\lim _{\varepsilon \rightarrow 0^{-}} D_{t}^{n-\varepsilon} f(t)=f^{(n)}(t)$,

(b) $\lim _{\varepsilon \rightarrow 0^{+}} D_{t}^{n-\varepsilon} f(t)=f^{(n)}(t)-f^{(n)}(0)$.

No entanto, existem algumas inconsistência tais como:

(1) Não obedece à familiar Regra do Produto para duas funções:

$$
D_{t}^{\alpha}[f(t) \cdot g(t)] \neq D_{t}^{\alpha} f(t) \cdot g(t)+f(t) \cdot D_{t}^{\alpha} g(t) .
$$

(2) Não obedeçe à Regra de Quociente para duas funções:

$$
D_{t}^{\alpha}\left[\frac{f(t)}{g(t)}\right] \neq \frac{g(t) D_{t}^{\alpha}(f(t))-f(x) D_{t}^{\alpha}(g(t))}{g^{2}(t)} .
$$

(3) Não obedece à Regra da Cadeia

$$
D_{t}^{\alpha}\left[f(g(t)] \neq D_{g(t)}^{\alpha} f(g(t)) \cdot D_{t}^{\alpha} g(t) .\right.
$$

Segundo Tarasov (2016) essas particularidades nos permitem descrever novos tipos incomus de sistemas físicos, naturais ou não. A violação da regra da cadeia é uma das principais características das derivadas fracionárias (SILVA, 2018).

Deve-se enfatizar que existe uma complicada regra da cadeia para o operador de Caputo, a Fórmula de Faà di Bruno (DIETHELM, 2010), sendo considerada uma limitação prática deste operador, e de outros operadores de ordem fracionária (TARASOV, 2016).

Neste trabalho, consideramos a seguinte equação diferencial fracionária envolvendo a derivada de Caputo:

$$
D_{t}^{\alpha} x(t)=f(t, x(t)), \quad \alpha \in(0,1),
$$

com a condição inicial $x_{0}=x(0)$.

Definição 2.4 A função de Mittag-Leffler de um parâmetro é dada por

$$
E_{\alpha}(t)=\sum_{k=0}^{\infty} \frac{t^{k}}{\Gamma(\alpha k+1)}
$$


Assim como a natureza da função exponencial é a solução de equações diferencias de ordem inteira, a função Mittag-Leffler tem um papel análogo para solução de muitas equações diferenciais de ordem não inteira. No início do século passado, Gösta Magnus Mittag-Leffler apresentou a função inteira

$$
E_{\alpha}(z)=\sum_{k=0}^{\infty} \frac{z^{n}}{\Gamma(\alpha k+1)}, \quad z \in \mathbb{C}, \Re(z)>0,
$$

que leva seu nome, e investigou suas propriedades básicas (MITTAG-LEFFLER, 1903, 1904, 1905). Para $0<\alpha \leq 1$, ela interpola a função exponencial e a função hipergeométrica $\frac{1}{1-z}$. É fácil ver que a função de Mittag-Leffler é uma generalização da função exponencial, pois quando $\alpha=1$ temos

$$
E_{1}(z)=\sum_{k=0}^{\infty} \frac{z^{k}}{\Gamma(k+1)}=\exp (z) .
$$

Além disso, para $\alpha \in(0,1)$, a restrição de $E_{\alpha}: \mathbb{R} \rightarrow \mathbb{R}$ é estritamente crescente, como observado em Cong, Son e Tuan (2014), Hilfer e Seybold (2006) e Pollard (1948) com

$$
\lim _{t \rightarrow+\infty} E_{\alpha}(t)=\infty \quad \text { e } \quad \lim _{t \rightarrow-\infty} E_{\alpha}(t)=0
$$

A função de Mittag-Leffler foi generalizada por Wiman (1905), que apresentou a função de Mittag-Leffler com dois parâmetros, $\alpha$ e $\beta$, da forma

$$
E_{\alpha, \beta}(z)=\sum_{k=0}^{\infty} \frac{z^{k}}{\Gamma(\alpha k+\beta)},
$$

com $\alpha, \beta \in \mathbb{C}, \mathfrak{R}(\alpha)>0$. Também é conhecida como função de Wiman. Estas funções permaneceram quase esquecidas durante mais de cinquentas anos, até que foram retomadas por Agarwal (1953), Humbert (1953), e vários outros.

Como caso especial, a função de Mittag-Leffler com um parâmetro é obtida para $\beta=1$, isto é, $E_{\alpha, 1}(z)=E_{\alpha}(z)$. Outra relação importante entre as funções Mittag-Leffler com um e dois parâmetros é dada por (PODLUBNY, 1999)

$$
E_{\alpha, \alpha+1}(z)=\frac{1}{z}\left[E_{\alpha}(z)-1\right]
$$

\subsection{Transformada de Laplace}

A transformada de Laplace é um conceito relevante para a resolução de equações envolvendo derivadas e integrais fracionárias. A seguir estão listadas algumas propriedades importantes para este trabalho. As demonstrações podem ser encontradas em Camargo e Oliveira (2015), Silva, Moreira e Moret (2018a):

$\mathrm{e}$

$$
\begin{gathered}
\mathscr{L}\left[D_{t}^{\alpha} f(t)\right]=s^{\alpha} F(s)-s^{\alpha-1} f(0), \quad 0<\alpha<1, \\
\mathscr{L}\left[E_{\alpha}\left(-\lambda t^{\alpha}\right)\right]=\frac{s^{\alpha-1}}{s^{\alpha}+\lambda}
\end{gathered}
$$

$$
\mathscr{L}^{-1}\left[\frac{s^{\alpha-\beta}}{s^{\alpha}+\lambda}\right]=t^{\beta-1} E_{\alpha, \beta}\left(-\lambda t^{\alpha}\right) .
$$




\section{Regra da cadeia de ordem $\alpha=1-\varepsilon, \operatorname{com} \varepsilon \ll 1$}

Considerando $n=1$ e $\alpha=1-\varepsilon$ na Equação (1) obtemos

$$
D_{t}^{1-\varepsilon} f(t)=\frac{1}{\Gamma(\varepsilon)} \int_{0}^{t} \frac{f^{\prime}(s)}{(s-t)^{1-\varepsilon}} d s
$$

Assumindo $f \in C^{2}[0, T]$ e aplicando o Teorema (2.3) para $t \in(0, T)$, obtemos

$$
\lim _{\varepsilon \rightarrow 0^{+}} D_{t}^{1-\varepsilon} f(t)=f^{\prime}(t)=\lim _{h \rightarrow 0} \frac{\Delta_{h} f(t)}{h},
$$

onde $\Delta_{\varepsilon} f(t)=f(t+\varepsilon)-f(t)$ denota o operador diferença progressiva.

Para a próxima definição precisaremos da seguinte equação $\lim _{h \rightarrow 0^{+}} \frac{\Delta_{h} f(t)}{h}=\lim _{h \rightarrow 0} \frac{\Delta_{h} f(t)}{h}=$ $\lim _{h \rightarrow 0^{-}} \frac{\Delta_{h} f(t)}{h}$, conhecida do cálculo clássico.

Assim, podemos escrever a equação (14) na forma

$$
\lim _{\varepsilon \rightarrow 0^{+}} D_{t}^{1-\varepsilon} f(t)=\lim _{\varepsilon \rightarrow 0^{+}} \frac{\Delta_{\varepsilon} f(t)}{\varepsilon}=\frac{\Delta_{\varepsilon} f(t)}{\varepsilon}+O(\varepsilon),
$$

em que $O(\varepsilon)$ é o erro de truncamento de ordem $\varepsilon(\varepsilon>0)$.

Dessa forma, a aproximação de primeira ordem para a derivada de Caputo com baixa fractalidade, $0<\varepsilon \ll 1$ :

$$
D_{t}^{1-\varepsilon} f(t) \simeq \frac{\Delta_{\varepsilon} f(t)}{\varepsilon}
$$

com erro de ordem $\varepsilon$.

As Fig. (1) e Fig. (2) exibem o gráfico das funções $f(t)=\mathscr{D}_{t}^{1-\varepsilon}\left(t^{4}\right)$ e $g(t)=\mathscr{D}_{t}^{1-\varepsilon}\left(e^{2 t}\right)$, utilizando Eq. (16), para $\varepsilon=0.01$. As soluções exatas são $\frac{\Gamma(5)}{\Gamma(4+\varepsilon)} t^{3+\varepsilon}$ e $2 t^{\varepsilon} E_{1,1+\varepsilon}(2 t)$, respectivamente. 
Figura 1: Solução exata versus solução aproximada da função $\mathscr{D}_{t}^{0.99}\left(t^{4}\right)$.

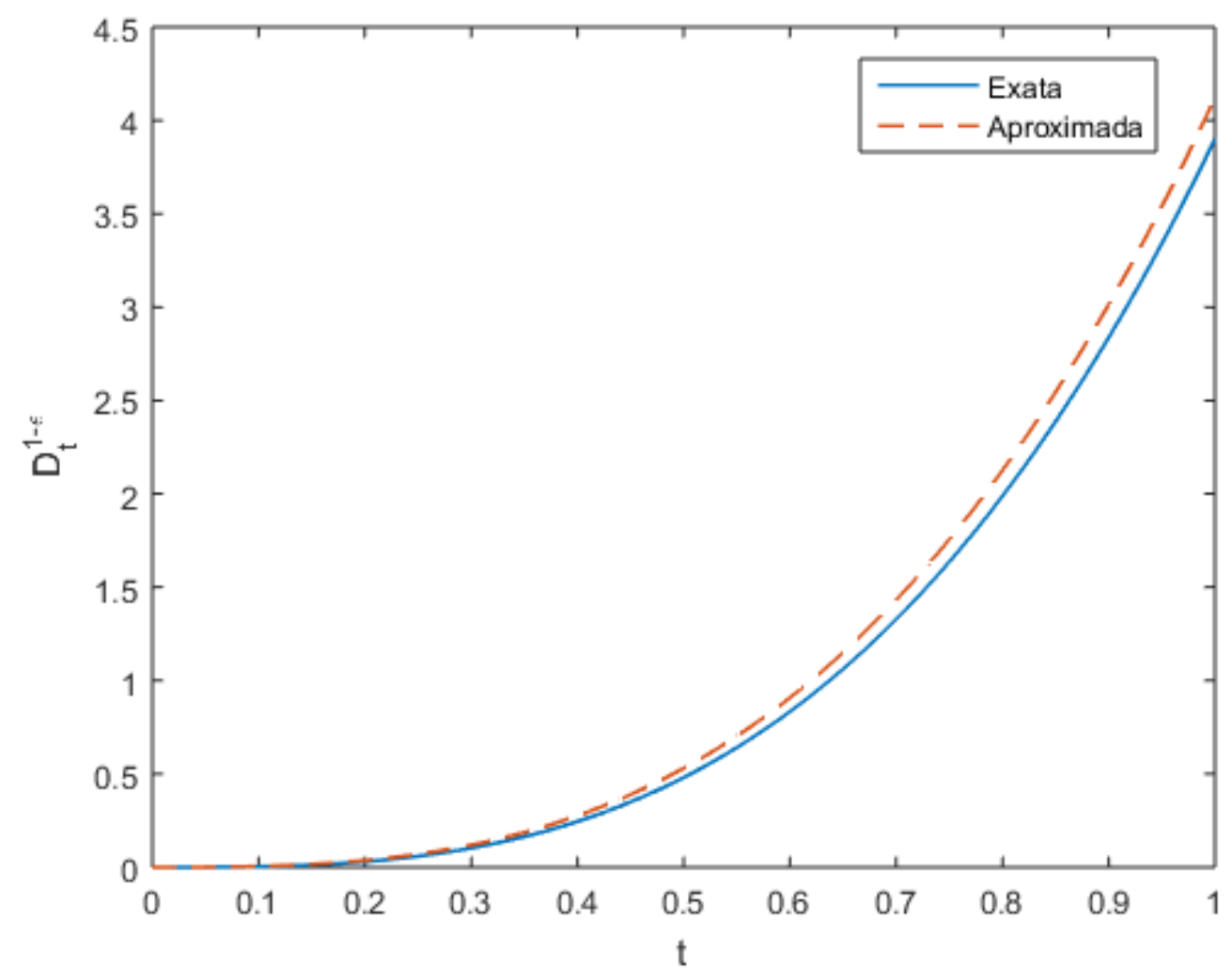

Esta aproximação de primeira ordem é válida para todos os operadores fracionários que possuem a propriedade da aproximação dada pelo Teorema (2.3).

Quando o incremento $\varepsilon$ é pequeno o suficiente, mas constante, podemos dizer que a diferença finita progressiva pelo incremento tende a derivada, mas não é igual a derivada de Caputo. Da mesma forma que o lado direito da Equação (16) também não é a derivada clássica.

Aplicando o Teorema (2.3) na função composta $f \circ g$, com $f$ e $g$ em $C^{2}[0, T]$, tem-se

$$
\begin{aligned}
\lim _{\varepsilon \rightarrow 0^{+}} D_{t}^{1-\varepsilon}[f \circ g](t) & =\left(D_{g}^{1} f(g)\right)_{g=g(t)} g^{\prime}(t), \\
& =\lim _{\varepsilon \rightarrow 0}\left[\frac{\Delta_{\varepsilon} f(g)}{\varepsilon}\right] \lim _{\varepsilon \rightarrow 0}\left[\frac{\Delta_{\varepsilon} g(t)}{\varepsilon}\right] .
\end{aligned}
$$

Imediatamente obtemos três aproximações possíveis combinando $D_{t}^{1-\varepsilon}$ ou $D_{t}^{1}$ na Equação (18) $\operatorname{com} \varepsilon \ll 1$ :

$$
\begin{aligned}
& D_{t}^{1-\varepsilon}[f \circ g](t) \simeq\left(D_{g}^{1} f(g)\right)_{g=g(t)} D_{t}^{1-\varepsilon} g(t) \\
& D_{t}^{1-\varepsilon}[f \circ g](t) \simeq\left(D_{g}^{1-\varepsilon} f(g)\right)_{g=g(t)} D_{t}^{1} g(t)
\end{aligned}
$$

$\mathrm{e}$

$$
D_{t}^{1-\varepsilon}[f \circ g](t) \simeq\left(D_{g}^{1-\varepsilon} f(g)\right)_{g=g(t)} D_{t}^{1-\varepsilon} g(t) .
$$

Escolhendo a Equação (19) como derivada de Caputo com baixo nível fracionário, $\alpha \simeq 1$, nós obtemos

$$
D_{t}^{\alpha}(f(g(t)))=\left(D_{g}^{1} f(g)\right)_{g=g(t)} D_{t}^{\alpha} g(t) .
$$


Figura 2: Solução exata versus solução aproximada da função $\mathscr{D}_{t}^{0.99}\left(e^{2 t}\right)$.

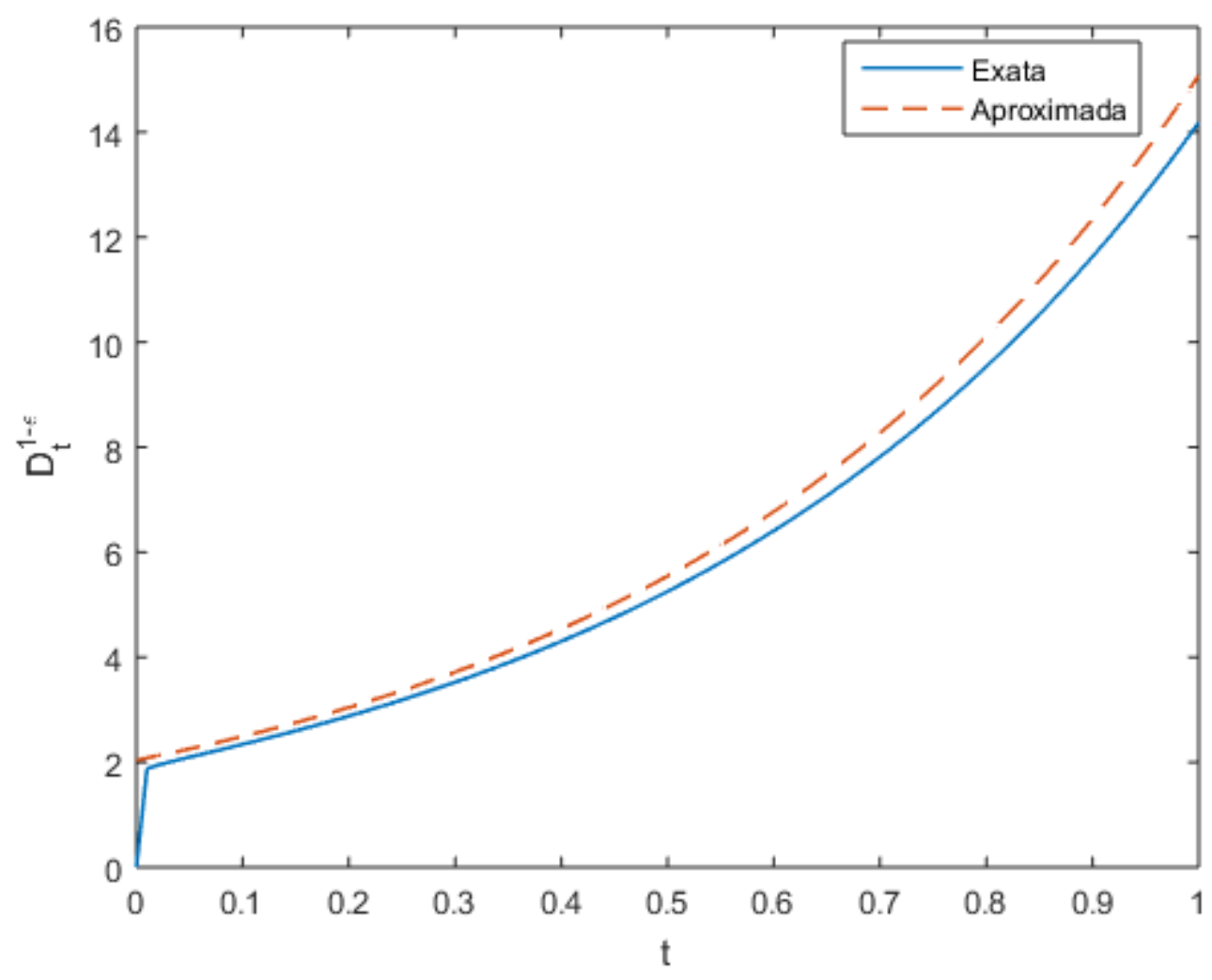

A regra da cadeia, derivada de ordem inteira, para a derivada de funções compostas dá origem ao chamado método da substituição ou de mudança de variável para resolver equações diferenciais. Esta técnica requer pouco cálculo e converte algumas equações diferenciais em tipos que podem ter suas soluções exatas conhecidas. Por exemplo, renomeando uma variável dependente para que $u(t)=y(z(t))$. Segue-se que

$$
D_{t}^{\alpha} u=y^{\prime}(z) D_{t}^{\alpha} z
$$

Com a escolha adequada de $z$, em situações especiais, a equação transformada (23) pode ser uma equação que possua solução exata conhecida.

\section{Resultados}

Usando a nova regra da cadeia para derivada de Caputo com baixo nível fracionário estudamos a equação diferencial fracionária dos tipos Bernoulli e o de Riccati. 


\subsection{Equações diferenciais fracionárias de Bernoulli com baixo nível fra- cionário}

Consideremos a versão de baixo nível fracionário para a clássica equação diferencial de Bernoulli com $0<\varepsilon \ll 1$, dada por:

$$
\left\{\begin{aligned}
D_{t}^{1-\varepsilon} y(t)+B(t) y(t) & =A(t) y^{q}(t) \\
y(0) & =y_{0} \geq 0,
\end{aligned}\right.
$$

onde $A(t)$ e $B(t)$ são funções contínuas e $q$ uma constante. Deve-se notar que algumas condições suficientes para a existência global de soluções da Equação (24) são dadas por Băleanu e Mustafa (2010) e Lakshmikantham, Leela e Devi (2009).

Para este fim, alteremos a variável dependente de $y$ para $u$ usando a seguinte substituição

$$
u(t)=[y(t)]^{1-q} .
$$

Consequentemente, em vista de (19) temos,

$$
D_{t}^{1-\varepsilon} u(t)=(1-q)[y(t)]^{-q} D_{t}^{1-\varepsilon} u(t) .
$$

Observe que se $q \neq 0$ e $q \neq 1$, a Equação (24) pode ser reduzida a uma forma linearizada

$$
D_{t}^{1-\varepsilon} u(t)+(1-q) B(t) u(t)=(1-q) A(t),
$$

cuja solução exata pode ser encontrada, em alguns casos, usando transformada de Laplace (ATANACKOVIC et al., 2014; CONG; SON; TUAN, 2014; LIANG; WU; CHEN, 2015).

Se para cada índice $\varepsilon, u_{\varepsilon}$ é uma solução da Equação (24) e se o limite

$$
y(t)=\lim _{\varepsilon \rightarrow 0^{+}}\left[u_{\varepsilon}(t)\right]^{\frac{1}{1-q}}
$$

existe para todo $t \in[0, T]$, então $y$ é solução do problema no caso $\varepsilon=0$, isto é, para equação de diferencial ordinária de Bernoulli.

Para encerrar esta seção apresentaremos dois exemplos. O último apresenta uma função exponencial deformada com dois parâmetros.

\subsubsection{Equações diferenciais fracionárias de Bertallanfy-Richards com baixo nível fracionário}

Considere a Eq. (24) $\operatorname{com} A(t) \equiv a, B(t) \equiv b$ e a condição inicial $y(0)=y_{0}>0$.

$$
D_{t}^{1-\varepsilon} y(t)=a y^{q}(t)-b y(t), \quad 0<\varepsilon \ll 1,
$$

onde $a \neq 0$ e $b \neq 0$ são constantes arbitrárias. Ao aplicar a mudança de variável conforme definido em (25), a Eq. (29) é tranformada em

$$
D_{t}^{1-\varepsilon} u=-(1-q) b u+(1-q) a .
$$

Aplicando a transformação de Laplace para ambos os lados, segue

$$
s^{1-\varepsilon} U(s)=s^{-\varepsilon} u(0)-(1-q) b U(s)+(1-q) b s^{-1} .
$$


Ou seja,

$$
u(t)=(1-q) a \mathscr{L}^{-1}\left[\frac{s^{-1}}{s^{1-\varepsilon}+(1-q) b}\right]+u(0) \mathscr{L}^{-1}\left[\frac{s^{-\varepsilon}}{s^{1-\varepsilon}+(1-q) b}\right]
$$

A partir disso, pela Eq. (12), temos:

$$
u(t)=(1-q) a t^{1-\varepsilon} E_{1-\varepsilon, 2-\varepsilon}\left[(q-1) b t^{1-\varepsilon}\right]+u_{0} E_{1-\varepsilon}\left[(q-1) b t^{1-\varepsilon}\right] .
$$

Então, usando a Eq. (9) e Eq. (25), de onde conclui-se

$$
y(t)=\left[\frac{a}{b}+\left(u_{0}^{1-q}-\frac{a}{b}\right) E_{1-\varepsilon}\left[(q-1) b t^{1-\varepsilon}\right]\right]^{\frac{1}{1-q}} .
$$

Se $(q-1) b<0$, da Eq. (7), a solução (34) converge para

$$
Y \equiv y(t \rightarrow \infty)=\left(\frac{a}{b}\right)^{\frac{1}{1-q}} .
$$

Contudo, como indicado em Varalta, Gomes e Camargo (2014) se $\varepsilon \rightarrow 0^{+}$então a função dada na Eq. (29) é equivalente a equação diferencial de Bertallanfy-Richards.

$$
y(t)=\left[\frac{a}{b}+\left(u_{0}^{1-q}-\frac{a}{b}\right) e^{b(q-1) t}\right]^{\frac{1}{1-q}}
$$

Exemplo 1 Em particular, para discutir o relaxamento não-linear Tofighi (2012) considerou $a=1, b=2$ e $y(0)=1$ na equação (29),

$$
D_{t}^{1-\varepsilon} y+2 y-y^{2}=0
$$

A solução da Eq. (37) com baixo nível fracionário é dada por

$$
y(t)=\frac{2}{1+E_{1-\varepsilon}\left(2 t^{1-\varepsilon}\right)} .
$$

\subsection{2 $(q, \alpha)$-Exponencial}

Considere Eq. (24) $\operatorname{com} A(t) \equiv a \neq 0, B(t) \equiv 0$ e a condição inicial $y(0)=1$.

$$
D_{t}^{\alpha} y(t)=a y^{q}(t)
$$

onde $a$ é uma constante arbitrária e $\alpha=1-\varepsilon$, com $0<\varepsilon \ll 1$. Ao aplicar a mudança de variável conforme definido em (25), a Eq. (39) é tranformada em

$$
D_{t}^{1-\varepsilon} u=(1-q) a
$$

Análogo a solução da Eq. (29), segue que

$$
y(t)=\left[1+a \frac{(1-q)}{\Gamma(1+\alpha)} t^{\alpha}\right]^{\frac{1}{1-q}} .
$$




\subsection{Equações diferenciais fracionárias de Riccati com baixo nível fracionário}

As equações diferenciais com baixo nível fracionário e do tipo Riccati são da forma

$$
\left\{\begin{aligned}
D_{t}^{1-\varepsilon} y(t)+B(t) y(t)+C(t) & =A(t) y^{2}(t) \\
y(0) & =y_{0} \geq 0 .
\end{aligned}\right.
$$

Se $C(t) \equiv 0$, a equação diferencial de Riccati reduz-se a um caso particular da equação diferencial fracionária de Bernoulli com $q=2$. No caso de $A(t) \equiv 0$, recai numa equação diferencial fracionária do tipo linear.

Se $A(t) \neq 0$ e conseguirmos de alguma forma obter uma solução particular da Eq. (42) $y_{p}$, então vale o seguinte resultado.

Teorema 4.1 Seja y uma solução particular da Equação (42). Então a substituição

$$
y(t)=y_{p}(t)+[z(t)]^{-1}
$$

transforma a equação diferencial fracionária de Riccati num equação diferencial linear fracionária na variável z

$$
D_{t}^{1-\varepsilon} z(t)+\left[2 A(t) y_{p}(t)-B(t)\right] z(t)=-A(t) .
$$

Demonstração: Da Eq. (43) obtém-se

$$
D_{t}^{1-\varepsilon} y(t)=D_{t}^{1-\varepsilon} y_{p}(t)-\frac{D_{t}^{1-\varepsilon} z(t)}{[z(t)]^{2}} .
$$

Substituindo em (42), obtemos

$$
D_{t}^{1-\varepsilon} y_{p}(t)+B(t) y_{p}(t)+C(t)-\frac{D_{t}^{1-\varepsilon} z(t)}{[z(t)]^{2}}+\frac{B(t)}{z(t)}=A(t)\left[y_{p}(t)\right]^{2}+\frac{2 A(t) y_{p}(t)}{z(t)}+\frac{A(t)}{[z(t)]^{2}} .
$$

Como $y_{p}$ é uma solução particular de (42), $D_{t}^{1-\varepsilon_{2}} y_{p}(t)+B(t) y_{p}(t)+C(t)=A(t)\left[y_{p}(t)\right]^{2}$ e, consequentemente, temos

$$
-\frac{D_{t}^{1-\varepsilon} z(t)}{[z(t)]^{2}}+\frac{B(t)}{z(t)}=\frac{2 A(t) y_{p}(t)}{z(t)}+\frac{A(t)}{[z(t)]^{2}} .
$$

Multiplicando Eq. (47) por $-[z(t)]^{2}$, obtemos a equação diferencial linear fracionária (44).

\subsubsection{Equação de Riccati com baixo nível fracionário e coeficientes constantes}

Considere a equação de Riccati (42) com coeficientes constantes e a condição inicial $u(0)=$ $u_{0}>0$, isto é,

$$
D_{t}^{1-\varepsilon} y(t)=a y^{2}(t)-b y(t)-c, \quad 0<\varepsilon \ll 1,
$$

onde $a \neq 0, b \neq 0$ e $c \neq 0$ são constantes arbitrárias.

Teorema 4.2 A Eq. (48) tem uma solução da forma $y=\chi$, sendo $\chi$ uma constante se, e somente se, $\chi$ é uma raiz da equação $a y^{2}-b y-c=0$.

O teorema acima permite, entre outras coisas, obter vários exemplos. 
Exemplo 2 Vamos considerar a equação diferencial fracionária de Riccati,

$$
D_{t}^{1-\varepsilon} y(t)=-[y(x)]^{2}+1, \quad 0<\varepsilon \ll 1,
$$

com condição inicial $y(0)=0$. Assim, pelo Teorema (4.2), $y_{p}(x) \equiv-1$ é uma solução particular da Eq. (49). A mudança de variável $y(t)=-1+\frac{1}{z(t)}$ transforma a Eq. (49) na equação diferencial

$$
D_{t}^{1-\varepsilon} z(t)+2 z(t)=1,
$$

com $z(0)=1$. Aplicando a metodologia acima obtemos a solução geral da Eq. (50) dada por

$$
z(t)=\frac{1}{2}\left(1+E_{1-\varepsilon}\left(-2 t^{1-\varepsilon}\right)\right)
$$

Finalmente, a solução geral da equação diferencial de Riccati é, assim, dada por

$$
\begin{gathered}
y(t)=-1+\frac{2}{1+E_{1-\varepsilon}\left(-2 t^{1-\varepsilon}\right)} \\
=\frac{1-E_{1-\varepsilon}\left(-2 t^{1-\varepsilon}\right)}{1+E_{1-\varepsilon}\left(-2 t^{1-\varepsilon}\right)}
\end{gathered}
$$

Ao aplicar o limite $\varepsilon \rightarrow 0^{+}$na Eq. (52), recuperamos o resultado clássico

$$
y(t)=\frac{e^{2 t}-1}{e^{2 t}+1} .
$$

\section{Conclusão}

A regra da cadeia com baixo nível fracionário justifica alguns versões fracionárias proposto por Varalta, Gomes e Camargo (2014) e Tavoni, Mancera e Camargo (2017). Acreditamos que nossa abordagem possibilitará a exploração novos caminhos e aplicações usando essa nova formulação da regra da cadeia. Além disso, esse método pode ser generalizado para outros tipos de Equações Diferenciais Fracionárias, o que será discutido em outros trabalhos.

\section{Agradecimentos}

Os autores agradecem aos revisores anônimos que contribuíram para a qualidade do artigo com seus comentários e sugestões.

\section{Referências bibliográficas}

AGARWAL, R. P. A propos d'une note de M. Pierre Humbert. Comptes Rendus de l'Academie des Sciences Paris, v. 236, n. 21, p. 2031-2032, 1953.

ATANACKOVIC, T. et al. Cauchy problems for some classes of linear fractional differential equations. Fractional Calculus and Applied Analysis, v. 17, n. 4, p. 1039-1059, 2014. 
BĂLEANU, D.; MUSTAFA, O. G. On the global existence of solutions to a class of fractional differential equations. Computers \& Mathematics with Applications, v. 59, n. 5, p. 1835$1841,2010$.

CAMARGO, R. F.; OLIVEIRA, E. C. Cálculo fracionário. São Paulo: Livraria da Física, 2015. CAPUTO, M. Linear models of dissipation whose $Q$ is almost frequency independent-II. Geophysical Journal International, v. 13, n. 5, p. 529-539, 1967.

CONG, N. D.; SON, D. T.; TUAN, H. T. On fractional Lyapunov exponent for solutions of linear fractional differential equations. Fractional Calculus and Applied Analysis, v. 17, n. 2, p. 285-306, 2014.

DIETHELM, K. The analysis of fractional differential equations: an application-oriented exposition using differential operators of caputo type. Berlin: Springer, 2010.

DU, M.; WANG, Z.; HU, H. Measuring memory with the order of fractional derivative. Scientific reports, v. 3, p. 3431, 2013.

EDELMAN, M. Fractional maps as maps with power-law memory. In: AFRAIMOVICH, A.; LUO, A. C. J.; FU, X. (Ed.). Nonlinear dynamics and complexity. [S.1.]: Springer, 2014. p. 79-120.

GOLDFAIN, E. Fractional dynamics and the standard model for particle physics. Communications in Nonlinear Science and Numerical Simulation, v. 13, n. 7, p. 1397-1404, 2008.

HILFER, R.; SEYBOLD, H. J. Computation of the generalized Mittag-Leffler function and its inverse in the complex plane. Integral Transforms and Special Functions, v. 17, n. 9, p. 637-652, 2006.

HUMBERT, P. Quelques résultats relatifs à la fonction de Mittag-Leffler. Comptes Rendus Hebdomadaires des Seances de L'Academie des Sciences, v. 236, n. 15, p. 1467-1468, 1953.

JUCHEM NETO, J. P.; FONSECA, C. N. Modelo de Solow com memória. In.: CONGRESSO NACIONAL DE MATEMÁTICA APLICADA E COMPUTACIONAL, 37., 2017, São José dos Campos. Proceedings... São José dos Campos: SBMAC, 2018.

LAKSHMIKANTHAM, V.; LEELA, S.; DEVI, J. V. Theory of fractional dynamical systems. Cambridge: Cambridge Scientific Publisher, 2009.

LI, C.; DENG, W. Remarks on fractional derivatives. Applied Mathematics and Computation, v. 187, n. 2, p. 777-784, 2007.

LIANG, S.; WU, R.; CHEN, L. Laplace transform of fractional order differential equations. Electronic Journal of Differential Equations, v. 2015, n. 139, p. 1-15, 2015.

MITTAG-LEFFLER, G. Sur la representation analytique d'une branche uniforme d'une fonction monogene. Acta Mathematica, v. 29, p. 101-181, 1905.

MITTAG-LEFFLER, G. M. Sur la nouvelle fonction $E_{\alpha}(x)$. Comptes Rendus de l'Academie des Sciences Paris, v. 137, n. 2, p. 554-558, 1903.

MITTAG-LEFFLER, G. M. Sopra la funzione $E_{\alpha}(x)$. Rendiconti della Reale Accademia dei Lincei (Ser. v), v. 5, n. 13, p. 3-5, 1904.

PODLUBNY, I. Fractional differential equations. San Diego: Academic Press, 1999. 
POLLARD, H. The completely monotonic character of the Mittag-Leffler function $E_{\alpha}(-x)$. Bulletin of the American Mathematical Society, v. 54, n. 12, p. 1115-1116, 1948.

SILVA, F. S. Conformable fractional integral equations of the second kind. Mathematica Aeterna, v. 8, n. 4, p. 199-205, 2018.

SILVA, F. S.; MOREIRA, D. M.; MORET, M. A. Conformable Laplace transform of fractional differential equations. Axioms, v. 7, n. 3, p. 55, 2018 a.

SILVA, F. S.; MOREIRA, D. M.; MORET, M. A. Regra da cadeia para derivada fracionária de Caputo com baixo nível fracionário. In.: ENCONTRO REGIONAL DE MATEMÁTICA APLICADA E COMPUTACIONAL, 5., Bauru. Cadernos de trabalhos completos e resumos..., Bauru: Unesp, Faculdade de Ciências, p. 255-260, 2018b. Disponível em: <https://drive.google. com/file/d/1-8SZZnioKApdmQG6BaOTltJARzfwAB9P/view?usp=sharing > . Acesso em: 21 set. 2018.

TARASOV, V. E. Review of some promising fractional physical models. International Journal of Modern Physics B, v. 27, n. 9, 2013.

TARASOV, V. E. On chain rule for fractional derivatives. Communications in Nonlinear Science and Numerical Simulation, v. 30, n. 1-3, p. 1-4, 2016.

TARASOV, V. E.; ZASLAVSKY, G. M. Dynamics with low-level fractionality. Physica A, v. 368, n. 2, p. 399-415, 2006.

TARASOVA, V. V.; TARASOV, V. E. Logistic map with memory from economic model. Chaos, Solitons \& Fractals, v. 95, p. 84-91, 2017.

TAVONI, R.; MANCERA, P. F. A.; CAMARGO, R. F. Modelos clássicos e fracionários de Gompertz e Bertalanffy. In.: CONGRESSO NACIONAL DE MATEMÁTICA APLICADA E COMPUTACIONAL, 37., 2017, São José dos Campos. Proceedings... São José dos Campos: SBMAC, 2017.

TOFIGHI, A. Nonlinear fractional relaxation. Pramana, v. 78, n. 4, p. 549-554, 2012.

TOFIGHI, A. An especial fractional oscillator. International Journal of Statistical Mechanics, v. 2013, 2013.

TOFIGHI, A.; GOLESTANI, A. A perturbative study of fractional relaxation phenomena. Physica A, n. 8-9, p. 1807-1817, 2008.

VARALTA, N.; GOMES, A. V.; CAMARGO, R. F. A prelude to the fractional calculus applied to tumor dynamic. TEMA, v. 15, n. 2, p. 211-221, 2014.

WIMAN, A. Über den fundamentalsatz in der teorie der funktionen $E_{\alpha}(x)$. Acta Mathematica, v. 29, n. 1, p. 191-201, 1905. 